\title{
MHD Casson fluid flow over a non-linear stretching/shrinking sheet with suction and slip boundary condition
}

\begin{abstract}
A problem of steady laminar magnetohydrodynamic (MHD) Casson flow over a non-linear stretching/shrinking sheet with suction and slip boundary condition is considered. The governing nonlinear partial differential equations are transformed into a system of nonlinear ordinary differential equations using a similarity transformation. The transformed ordinary differential equations are then solved numerically using the bvp4c function in MATLAB software. Dual solutions are found for a certain range of the governing parameter. The effects of the suction parameter and the slip boundary condition on the skin friction and heat transfer coefficients as well as the velocity and temperature profiles are presented and discussed.
\end{abstract}

Keyword: MHD; Casson; Stretching/shrinking; Dual solutions; Slip 